\title{
The tale of two vessels, vascular complications following a breach of the pelvic inner table due to acetabular screws: a report of two cases
}

\author{
Khairul Anwar Ayob, MBBS MS'ㄹ, Azhar M. Merican, MBBS MS PhD'10, \\ Shahrul-Hisham Sulaiman, MBBS MS $^{2}$ (D) Ahmad Rafizi Hariz Ramli, MD MS FEBVS ${ }^{3}$ [D \\ 'Department of Orthopaedic Surgery (NOCERAL), Faculty of Medicine, University Malaya, Kuala Lumpur, Malaysia \\ ${ }^{2}$ Department of Orthopaedic, Faculty of Medicine, Universiti Teknologi MARA, Sungai Buloh, Selangor, Malaysia \\ ${ }^{3}$ Department of Surgery, Faculty of Medicine, University Malaya, Kuala Lumpur, Malaysia
}

Vascular injury arising from perforation of the inner table due to acetabular screw drilling or fixation is a rare occurrence. ${ }^{[1]}$ However, the consequences are often dire and there must be a high index of suspicion as the diagnosis and treatment can be delayed, invariably leading to significant morbidity or even mortality. By studying cadaveric pelves, Wasielewski et al. ${ }^{[2]}$ devised an intraoperative method of dividing the acetabulum into four quadrants as a simple guide for intra-acetabular screws for cementless cup placement. The superoposterior and inferoposterior quadrants are described as safe zones, with the best bone stock for optimal fixation. In this article, we report two cases of vascular complications as a result of screw placement to optimize fixation of acetabular

Received: July 03, 2020

Accepted: October 02, 2020

Published online: January 06, 2021

Correspondence: Khairul Anwar Ayob, MBBS. Department of Orthopaedic Surgery (NOCERAL), Faculty of Medicine, University Malaya, 50603 Kuala Lumpur, Malaysia.

E-mail: khairul.ayob@ummc.edu.my

Doi: $10.5606 /$ ehc. 2021.77862

Citation: Ayob KA, Merican AM, Sulaiman SH, Hariz Ramli AR. The tale of two vessels, vascular complications following a breach of the pelvic inner table due to acetabular screws: a report of two cases. Jt Dis Relat Surg 2021;32(1):239-244.

\section{(C2021 All right reserved by the Turkish Joint Diseases Foundation}

This is an open access article under the terms of the Creative Commons Attribution-NonCommercial License, which permits use, distribution and reproduction in any medium, provided the original work is properly cited and is not used for commercial purposes (http://creativecommons.org/licenses/by-nc/4.0/).

\section{ABSTRACT}

Injuries to the pelvic vasculature during total hip arthroplasties are rare but have serious consequence. They demand urgent and early identification so that appropriate treatment can be instituted. If the bleeding is severe, cardiovascular compromise occurs intraoperatively and this will alert the surgeon of this possibility during acetabular screw placement. Alternatively, a delay in diagnosis can occur because the bleeding and the injured vessel are in the pelvic cavity and not visualized during the surgery. In this article, we report two cases from our center occurring within a six-month interval that sustained a vascular injury during acetabular drilling for screw placement for cementless cup fixation. Each case had a different vessel injury and different lessons can be learned from these rare injuries. The first case had an injury of the inferior gluteal artery following a breach of the sciatic notch. The vessel was treated with percutaneous embolization. The second case demonstrated a venous injury, following a medial protrusio technique for congenital hip dysplasia and a short anterosuperior screw, transecting the external iliac vein. This was subsequently repaired using an endovascular technique. We conclude the reasons for these vessel injuries after analyzing advanced imaging, discuss measures to avoid vessel injury and detail the minimally invasive method for their treatment.

Keywords: Case report, complications, external iliac vein, inferior gluteal artery, total hip arthroplasty, vascular injury.

cups. One case required intravascular stenting of the affected vessel, while the other required embolization.

\section{CASE REPORT}

Case 1- A 64-year-old male patient (height $170 \mathrm{~cm}$ ) was scheduled for a revision right total hip arthroplasty (THA). He was initially referred for a prosthetic joint infection, which was managed with debridement, removal of implants and antibiotic cement spacer for his right hip. There was no history of peripheral 
vascular disease and pulses were palpable for both lower limbs. Access was through a posterior approach, and the acetabulum was prepared for the Trident ${ }^{\circledR}$ Acetabular System (Stryker, Kalamazoo, MI, USA). After a $52 \mathrm{~mm}$ cementless cup was placed, a $35 \mathrm{~mm}$ screw was inserted at the posterosuperior quadrant aspect of the cup, and this was uneventful. A second more posterior screw hole was then drilled, after which we noticed a continuous pulsatile backflow from the screw hole. The screw hole length measured $40 \mathrm{~mm}$ and palpation with the depth gauge confirmed solid bone at the end of the screw hole. We also noticed an acute drop in systolic blood pressure (SBP) to $80 \mathrm{mmHg}$ during this event. The SBP then normalized to above $100 \mathrm{mmHg}$ during a second reading $5 \mathrm{~min}$ later. The heart rate remained below $100 \mathrm{bpm}$ during the surgery. The screw hole continued to have pulsatile backflow after $5 \mathrm{~min}$, so no screw was inserted and the screw hole was left empty. The surgery was completed expediently, and we sought assistance from our vascular surgery colleagues. The patient was then repositioned supine and an on-table angiogram was performed, gaining access through the contralateral femoral artery, which demonstrated an injury to the inferior gluteal artery (IGA) (Figure 1). The IGA was then embolized, deploying three coils (sized $0.035^{\prime \prime} \times 5 \mathrm{~mm}, 0.035^{\prime \prime} \times 5 \mathrm{~mm}$ and $0.035^{\prime \prime}$ $\times 6 \mathrm{~mm}$ ). The patient remained stable throughout the procedure. His recovery was uneventful. Follow-ups after 14 months revealed no complications and the patient reported being satisfied with the surgery.

Critical three-dimensional (3D) analysis of the postoperative computed tomography (CT) scan showed that the posterior screw tract breached the inner table at a relatively short distance (Figure 2). However, the drill hole extended into the ilium adjacent to the sacrum, which gave a false sense of the length of the screw. A written informed consent was obtained from the patient.

Case 2- Six months after Case 1, a 55-year-old female patient (height $148 \mathrm{~cm}$ ) was scheduled for a THA. Preoperatively, she was diagnosed with a neglected developmental dysplasia of the hip with a high riding, dislocated femoral head (Crowe 4). There was no history of previous venous thromboembolism disease, and there were no signs of chronic venous insufficiency. A total hip replacement was performed using a posterior approach, involving a femoral osteotomy to shorten and derotate the femur. An S-ROM ${ }^{\circledR}$ modular prosthesis was used for the femur, while a $44 \mathrm{~mm}$ Pinnacle ${ }^{\circledR}$ Bantam titanium cup was used for the acetabulum (both components from Johnson \& Johnson, Warsaw, IN, USA). As the acetabulum was severely dysplastic with poor bone stock, a medial protrusio technique was performed to allow for better coverage of the acetabular cup. One screw was inserted in the posterior superior quadrant. During the drilling of the second screw at the superior aspect, an instantaneous gush of blood flowed out from the screw hole. There was no further bleeding and the patient remained hemodynamically stable throughout the surgery. A $20 \mathrm{~mm}$ screw was then placed. The rest of the surgery was uneventful.

During the recovery period, the patient developed a progressive swelling of the operated limb. Calf circumference monitoring at postoperative
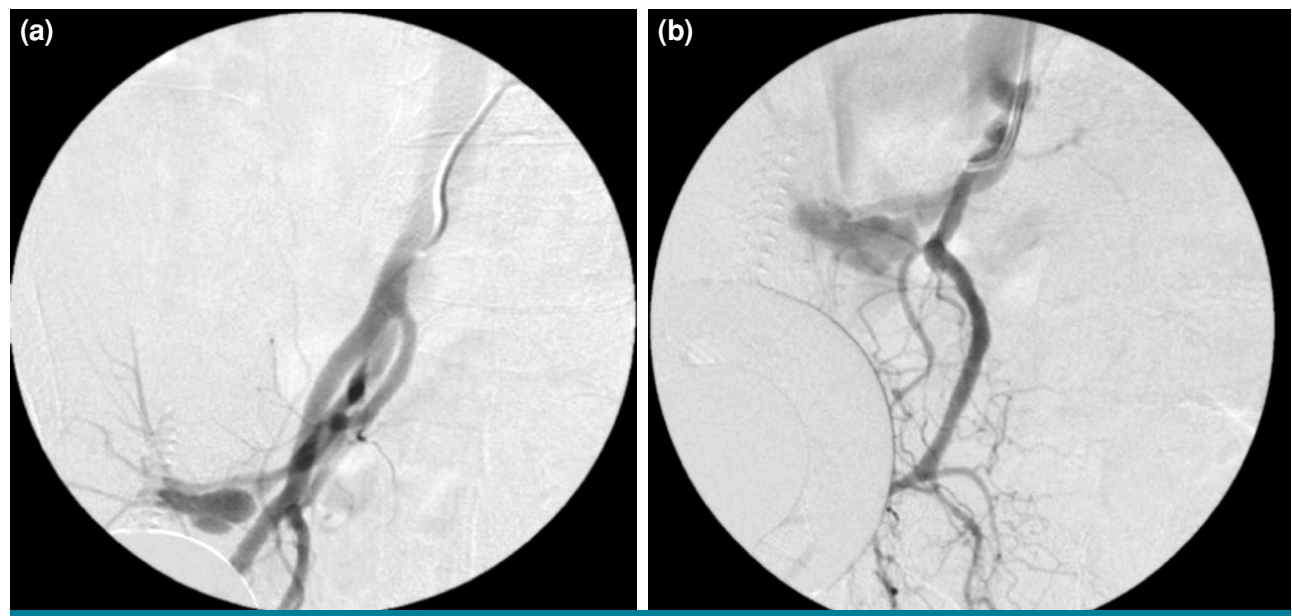

FIGURE 1. Angiography findings of internal iliac artery in Case 1. (a) Extravasation of contrast noted from inferior gluteal artery, silhouette of implants noted nearby. (b) Coiling of inferior gluteal artery. 
(a)

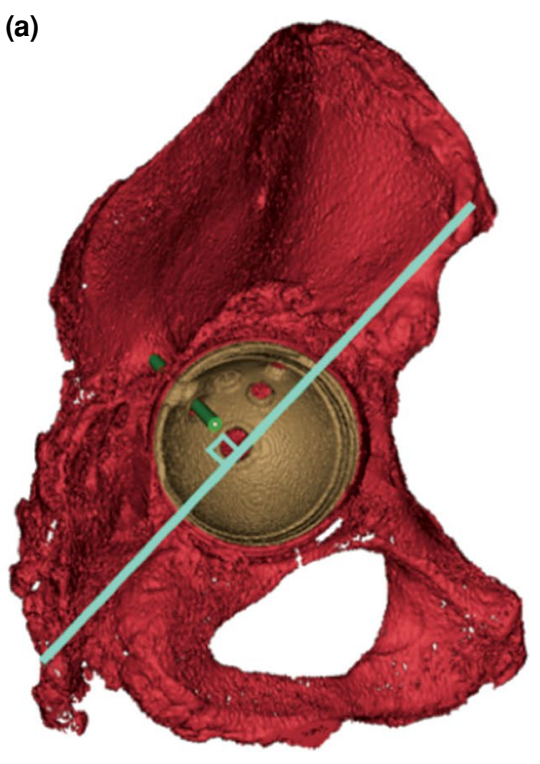

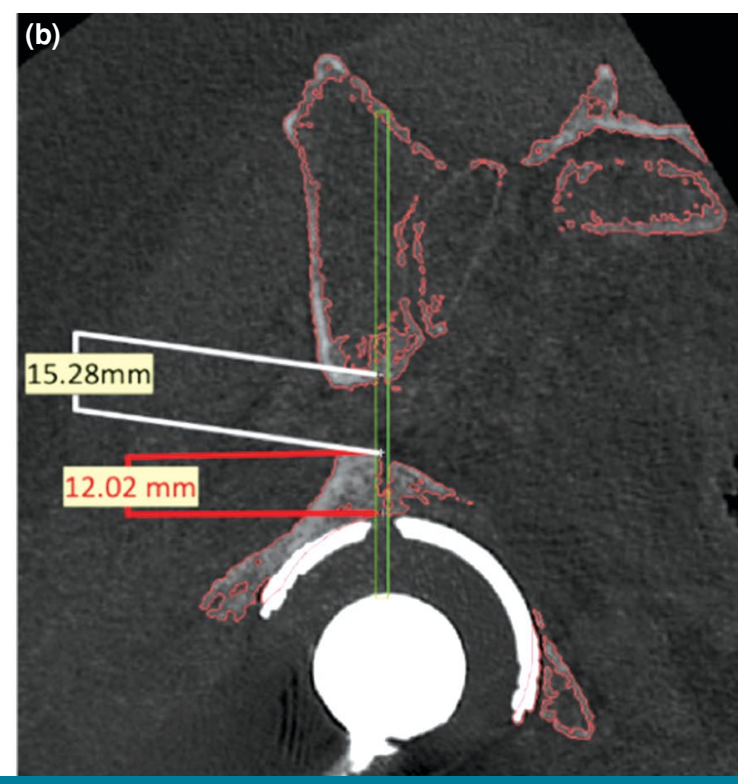

FIGURE 2. (a) Three-dimensional reconstructed computed tomography imaging of pelvis and cup, showing posterior screw tract (green) breaching inner table at sciatic notch, and re-entering ilium adjacent to sacrum. (b) Computed tomography was recut according to screw tract, shows length of bone at area of only $12 \mathrm{~mm}$ before table is breached and notch violated. However, tract does re-enter bone, and this may give a false sense of security to surgeon.

$48^{\text {th }} \mathrm{h}$ revealed a $5 \mathrm{~cm}$ increase compared to the contralateral side. Her peripheral pulses remained palpable. Postoperative radiographs revealed that the acetabular screw had penetrated the inner table. A Doppler ultrasound confirmed a long segment thrombosis starting at the left popliteal vein, extending to the left external iliac vein. She was referred to our vascular colleagues and a CT venogram was requested, which confirmed a hematoma deep to the acetabular cup. The external iliac vein (EIV) was non-opacified while its tributaries, the common femoral vein and the superficial femoral vein were dilated with hypodensity, likely from developing a thrombus.

Due to the presence of venous thromboembolism, an inferior vena cava filter was placed, at which point a percutaneous repair was performed. A venogram was performed via access from the left popliteal vein, which identified the distal aspect of the EIV. The contralateral right popliteal vein was then accessed to identify the proximal stump. The venogram showed that the left EIV was completely transected with distal EIV thrombosis. Aspiration thrombectomy was performed from popliteal vein to left EIV. The left EIV was then reconnected with a 10x80 mm Fluency ${ }^{\circledR}$ vascular graft stent (Bard Peripheral Vascular, Inc., Tempe, AZ, USA). Subsequent venograms showed reconstitution of the flow of the left EIV. Following revascularization of the EIV, the patient's lower limb swelling progressively reduced in size and her subsequent postoperative rehabilitation was uneventful. Follow-up at eight months confirmed that her venous circulation remained intact and she was satisfied with the results of her THA. A written informed consent was obtained from the patient.

\section{DISCUSSION}

These cases demonstrate the possible complications of employing screws for improved fixation of a cementless cup in THA. ${ }^{[3]}$ The risk of pelvic vasculature injuries is reported to be low, between 0.06 to $0.25 \% .{ }^{[4]}$ Nevertheless, vascular injuries can lead to serious consequences and hence a high index of suspicion is mandated to identify such injuries. Delayed recognition is highly associated with significant morbidity, and may lead to sudden or eventual death. ${ }^{[5]}$

The external iliac, obturator, superior and inferior gluteal, and internal pudendal arteries and veins are intrapelvic and particularly at risk when breaching the inner cortex of the pelvis. Regrettably, the visualization of said structures is impossible intraoperatively. Thus the course and location of these vessels have been well described and safe zones have 

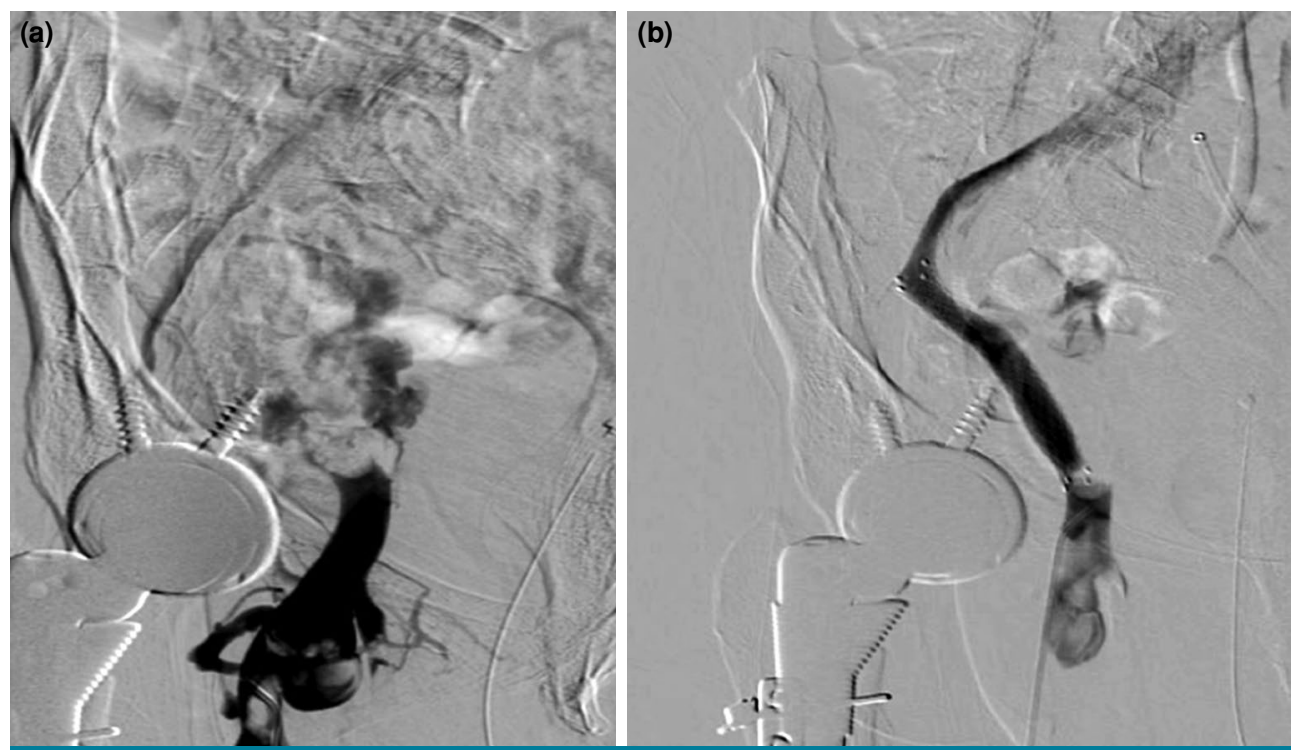

FIGURE 3. Venography findings from before and after endovascular repair of external iliac vein in Case 2. (a) Venogram showing occlusion of distal part of external iliac vein with mild contrast extravasation. (b) Venogram after deployment of $10 \times 80 \mathrm{~mm}$ Fluency ${ }^{\circledR}$ stent, which confirmed blood flow through external iliac vein.

been described using visible structures on the outer aspect of the pelvis to avoid injuring these vessels. ${ }^{[2,6]}$

Particularly in complex primary or revision THAs, a stable cementless cup is desirable to encourage osteointegration to the implant and ensure the longevity of the prosthesis. Good initial stability is best supplemented with transcortical screws ${ }^{[7]}$ as opposed to press-fit fixation or unicortical cancellous screws. However, this intraoperative decision needs to balance the risk of injuring the deep pelvic vessels when breaching the inner cortex of the acetabulum. In our first case, the screw was inserted according to the recommended safe zone, but unfortunately, the drill breached the sciatic notch $12 \mathrm{~mm}$ from the entry point, where the IGA was injured. The distance from the cup $(12 \mathrm{~mm})$ to the injured vessel in Case 1 is demonstrated in Figure 3. It then re-entered the pelvis and made it seem like the screw tract length was longer than it truly is. Identification was prompt
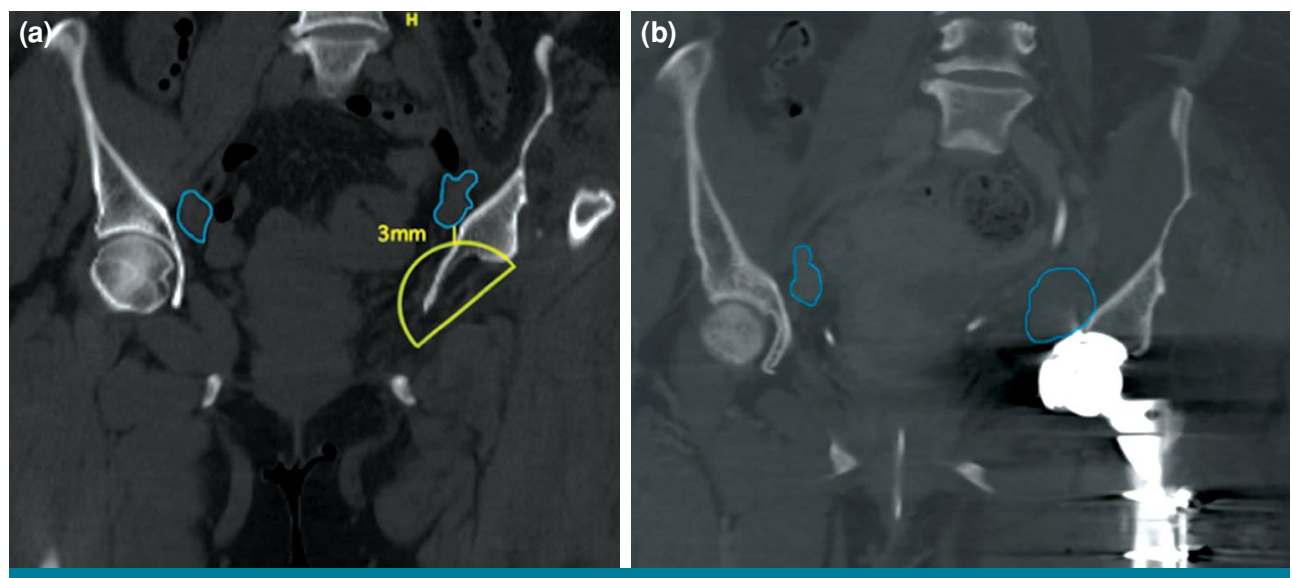

FIGURE 4. Computed tomography scan findings from before and after THA. (a) Preoperative CT with both EIVs outlined. Planned position of cup is demonstrated, and its proximity to EIV measured. (b) Computed tomography findings after THA, showing position of acetabular cup and formation of a hematoma at EIV site on left and right EIV on non-operated site (outlined).

THA: Total hip arthroplast; CT: Computed tomography; EIV: External iliac vein 
from the pulsatile bleeding and transient drop in systolic blood pressure. The course of the IGA has been studied in the Caucasian and Asian populations and although the posterosuperior quadrant has been described to be relatively safe, the surgeon needs to be wary of the length of the drill or the screw, particularly in proximity to the sciatic notch, where even a short screw may put the gluteal vessels at risk. ${ }^{[1,2]}$ The reported incidence of IGA injury is rare, we only identified two cases from previously published case series by Mavrogenis et al. ${ }^{[8]}$ and Cheng et al., ${ }^{[9]}$ both occurring as a complication from THA and treated with embolization.

Particularly when inserting screws posteriorly, surgeons should be aware of the feel of resistance and subsequent give when drilling, taking care not to plunge too deep. In a degenerative hip, the subchondral bone can be dense and excessive force can cause the drill to suddenly perforate the far cortex and beyond. This is particularly the case when the drill is not sharp. Moreover, in acetabular drilling, a flexible or offset drill bit is often required, and this can require more force and less control in-depth penetration. Ensuring that the drill bit is sharp has been shown to improve the feel and reduce the plunge depth of the drill bit and reduce the risk of deep structure injuries. ${ }^{[10]}$ Palpation of the sciatic notch is another strategy in guiding surgeons regarding the best direction for the drill to avoid breaching the notch. ${ }^{[11]}$ A high index of suspicion may be beneficial for early identification intraoperatively, as is good communication with the anesthetist. Early on-table referral to vascular or surgical colleagues and close monitoring in the intensive care unit can improve patient outcomes. ${ }^{[12]}$

Hemorrhage control is essential in the treatment of these patients and best achieved through percutaneous methods; ${ }^{[13]}$ however, in the absence of intravascular interventional expertise, open exploration and ligation may be considered in patients who deteriorate despite optimal resuscitation measures.

In our second case, the true acetabulum was deformed and shallow. Therefore, to place the cup at the true hip center, a cotyloplasty was employed to medialize the cup and subsequently improve the bony coverage. Unfortunately, the screw hole was hence positioned nearer to the EIV, which was inadvertently injured. This occurred despite utilizing a short drill and screw for fixation of the cup. In Case 2, the distance of bone available for screw fixation was shorter than $15 \mathrm{~mm}$. As demonstrated in Figure 4, the pre- and postoperative
CT scan demonstrated the proximity of the EIV to the true acetabulum. We were also restricted by the smaller Bantam cup, which only has six screw hole options for the screw fixation, each separated by about 60 degrees. Other, larger cups can provide more screw direction options, which may be directed to avoid the vessel.

Fortunately for the patient, the distal stump of the EIV contracted and a thrombus was formed rapidly, preventing catastrophic blood loss. We hypothesize that the total transection allows for the contraction of smooth muscles at the ends, stopping the bleeding and then an occluding clot form. In contrast, in a partial laceration, contraction of the smooth muscle may enlarge the opening and worsen the blood loss. This phenomenon has been described for arterial injuries ${ }^{[14]}$ as arteries have a thicker and more well-defined tunica media. Our literature review revealed two cases whereupon the EIV was injured from orthopedic procedures involving a breach of the inner cortex of the acetabulum. Müdüroglu et al. ${ }^{[15]}$ described a case very similar to ours, where an EIV injury occurred during screw placement for an acetabular cup in a dysplastic hip. Due to postoperative hemodynamic instability, an emergent abdominal exploration and packing was performed, in addition to a second stage open venous grafting. A similar injury to the EIV was described by Siegel et al. ${ }^{[16]}$ following breaching of the inner acetabular cortex by a guide wire when performing a dynamic hip screw for an intertrochanteric fracture. Their case was stable in the intraoperative period but deteriorated during the immediate postoperative period. Unfortunately, their patient suffered from circulatory collapse, which eventually was non-responsive to resuscitation.

Our patient continued to be hemodynamically stable but then developed symptoms of venous congestion due to incompetent venous drainage of the affected limb. This could be mistaken for a deep vein thrombosis; however, a CT venogram was ordered given the incidence of bleeding from the screw hole, and the proud acetabular screw demonstrable from radiographs.

Repair of the vein was performed endovascularly and this has been proven to be a safe, durable and effective measure for addressing iliac vein ruptures. An open repair has higher morbidity and mortality rates and conservative treatment is preferred compared to open repair. ${ }^{[10]}$ This method limits the additional soft tissue trauma and risks associated with open exploration and repair soon after a hip replacement. 
To obtain a diagnosis, a CT angiogram (CT with contrast administered through a peripheral venous line) can also be employed. During the venous phase, a complete venous obstruction can also be visualized. This is readily available in most hospitals. When the diagnosis is established, a vascular surgeon or an interventional radiologist, if available, can perform either a percutaneous reconstruction or embolize the vessel by performing an interventional angiogram. Although open repair was previously performed for venous injuries in the absence of angiography availability, it is highly associated with significant limb and wound morbidities. ${ }^{[13]}$

Although rare, vascular complications following total hip replacement can be devastating. A comprehensive understanding of the courses of different vascular structures and the safe zones for screw placement is crucial, not to mention awareness of the depth of the bone in different areas. Always ensure that the drill bit is sharp prior to drilling acetabular screws. A CT scan of the pelvis and 3D reconstruction for preoperative planning of complex primary or revision cases should be considered as apart from looking at bony anatomy and defects, it is useful to identify positions of important structures and employ tactics to avoid such injuries. The availability of expertise and equipment to further assess and manage these complications is desirable, particularly in anticipation of complex cases and in centers which have a high volume of such cases. Keep in mind that the bleeding may not be dramatic so surgeons can be lulled into a false sense of security. For this reason too, it is likely that vessel injuries can be underreported.

Our experience underscores the fact that particularly in cases where cups need to be placed in non-anatomic positions, there are no true safe zones for cementless acetabular screw placement. Some are less dangerous than others.

\section{Declaration of conflicting interests}

The authors declared no conflicts of interest with respect to the authorship and/or publication of this article.

\section{Funding}

The authors received no financial support for the research and/or authorship of this article.

\section{REFERENCES}

1. Ohashi H, Kikuchi S, Aota S, Hakozaki M, Konno S. Surgical anatomy of the pelvic vasculature, with particular reference to acetabular screw fixation in cementless total hip arthroplasty in Asian population. J Orthop Surg (Hong Kong) 2017;25:2309499016685520.

2. Wasielewski RC, Cooperstein LA, Kruger MP, Rubash HE. Acetabular anatomy and the transacetabular fixation of screws in total hip arthroplasty. J Bone Joint Surg [Am] 1990;72:501-8.

3. Barrack RL, Butler RA. Avoidance and management of neurovascular injuries in total hip arthroplasty. Instr Course Lect 2003;52:267-74.

4. Alshameeri Z, Bajekal R, Varty K, Khanduja V. Iatrogenic vascular injuries during arthroplasty of the hip. Bone Joint J 2015;97-B:1447-55.

5. Atik OŞ. What are the expectations of an editor from a scientific article? Jt Dis Relat Surg 2020;31:597-8.

6. Harkess, JW, Crockarell, JR. Arthroplasty of the hip. In: Canale ST, Azar FM, Beaty JH, editors. Campbell's Operative Orthopaedics. 13th ed. Philadelphia, PA: Elsevier/ Mosby; 2017. p. $165-321$

7. Burkhart TA, Khadem M, Wood TJ, Dunning CE, Degen R, Lanting BA. Comparison of trans-cortical and cancellous screws to press fit for acetabular shell fixation in total hip arthroplasty: A cadaveric study. Clin Biomech (Bristol, Avon) 2019;69:34-8.

8. Mavrogenis AF, Rossi G, Rimondi E, Ruggieri P, Mercuri M. Embolisation for vascular injuries complicating elective orthopaedic surgery. Eur J Vasc Endovasc Surg 2011;42:676-83.

9. Cheng H, Shin JH, Yoon HK, Choe J, Ko GY, Gwon DI, et al. Retrospective 12-year study of the safety and efficacy of transcatheter arterial embolization for managing bleeding complications following hip surgery. Cardiovasc Intervent Radiol 2014;37:1464-8.

10. Alajmo G, Schlegel U, Gueorguiev B, Matthys R, Gautier E. Plunging when drilling: effect of using blunt drill bits. J Orthop Trauma 2012;26:482-7.

11. Wasielewski RC, Kusuma S, Rosenberg AG. Gross anatomy of the hip. In: Callaghan JJ, Rosenberg AG, Rubash HE, Clohisy JC, Beaulé PE, Della Valle CJ, editors. The adult hip: Hip arthroplasty surgery. Vol 1. 3rd ed. Philadelphia: Wolters Kluwer Health Adis (ESP) 2015. p. 37-54.

12. Calligaro KD, Dougherty MJ, Ryan S, Booth RE. Acute arterial complications associated with total hip and knee arthroplasty. J Vasc Surg 2003;38:1170-7.

13. Troutman DA, Dougherty MJ, Spivack AI, Calligaro KD. Updated strategies to treat acute arterial complications associated with total knee and hip arthroplasty. J Vasc Surg 2013;58:1037-42.

14. Edwards EA, Fiddian RV. Blood vessel injury: Features of the pathology. Postgrad Med J 1963;39:724-8.

15. Müdüroğlu A, Kayhan TO, Yüksel A. Iliac vein injury during total hip replacement: a rare iatrogenic complication and its successful surgical treatment. Int J Vasc Surg Med 2017;3:33-5.

16. Siegel A, Schulz F, Püschel K. Fatal pelvic vein injury caused by guidewire used with the dynamic hip screw. Unfallchirurg 2001;104:182-6. 